\title{
Finite size corrections to the spectrum of regular random graphs: an analytical solution
}

\author{
F. L. Metz ${ }^{1}$, G. Parisi ${ }^{1,2,3}$ and L. Leuzzi ${ }^{1,2}$ \\ 1 Dip. Fisica, Università La Sapienza, Piazzale A. Moro 2, I-00185, Rome, Italy \\ 2 IPCF-CNR, UOS Roma Kerberos, Università La Sapienza, Piazzale A. Moro 2, I-00185, Rome, Italy \\ 3 INFN, Piazzale A. Moro 2, 00185, Rome, Italy
}

( $\Omega$ Dated: June 12, 2018)

\begin{abstract}
We develop a thorough analytical study of the $O(1 / N)$ correction to the spectrum of regular random graphs with $N \rightarrow \infty$ nodes. The finite size fluctuations of the resolvent are given in terms of a weighted series over the contributions coming from loops of all possible lengths, from which we obtain the isolated eigenvalue as well as an analytical expression for the $O(1 / N)$ correction to the continuous part of the spectrum. The comparison between this analytical formula and direct diagonalization results exhibits an excellent agreement, confirming the correctness of our expression.
\end{abstract}

PACS numbers: 05.40.-a, 89.75.Hc,71.23.-k

\section{INTRODUCTION}

Spectral graph theory has established itself as a fundamental tool to study problems in various disciplines [1]. On the side of physics, the understanding of stationary and dynamical properties of models defined on random graphs depends crucially on the spectral analysis of the adjacency and the Laplacian matrix of the corresponding graph. The average distribution of eigenvalues constitutes a primary object of interest, due to its wide range of applications. Some notable examples include the study of the vibrational spectra of amorphous solids [2], the electronic properties of quantum systems [3] and spherical spin models [4].

A central role in spectral graph theory is played by sparse random regular graphs (RRGs), since they constitute a benchmark for analyzing the spectral features of more complex graph structures. Random regular graphs are constructed by drawing, from an uniform probability space, simple undirected graphs where all vertices have the same degree. Sparse RRGs become locally treelike when the total number of nodes $N$ grows to infinite, such that only long loops of length $O(\ln N)$ are present. Thanks to the absence of degree fluctuations and to the local tree-like structure, many spectral properties of RRGs can be analytically studied using non-rigorous [5, 6] as well as rigorous mathematical approaches (see 7] and references therein). In this context, the most prominent example is the average eigenvalue distribution of the adjacency matrix, which converges, for $N \rightarrow \infty$, to a simple analytical expression known as the Kesten-McKay (KM) law [8, 9].

Much less is known about the finite size fluctuations of the spectra of sparse random graphs. The existence of short loops on graphs with a finite size and the impact of these topological fluctuations on the spectral properties is an interesting problem on its own right. In addition, sparse random graph models usually lead, due to its local tree-like topology, to a mean-field description of models defined on finite-dimensional lattices and, in a certain sense, the construction of a perturbative expansion in powers of $O(1 / N)$ for random graph models constitutes an indirect route to study the intricate role of loops on their finite-dimensional counterparts. In fact, analogous ideas have been put forward in the context of Anderson localization and statistical mechanics of spin systems 10 13], where the behavior of models defined on finitedimensional lattices is studied perturbatively around the mean-field saddle-point corresponding to sparse random graph models.

Here we implement these ideas to study the $O(1 / N)$ correction to the average eigenvalue distribution of the adjacency matrix of RRGs, which are simple enough to render a full analytical study possible. We show that the $O(1 / N)$ correction to the resolvent of the adjacency matrix is given by a sum over loops comprising all length scales, each loop contributing with a term proportional to the difference of its effective resolvent with respect to the resolvent of an infinite closed chain. Within the replica approach for random matrices [14, 15], this result is derived from an integration of the $O(1 / \sqrt{N})$ fluctuations of a functional order-parameter around its saddle-point solution, following analogous steps as those developed recently to the study of finite size corrections of models with quenched disorder [16, 17]. We show how the divergent loop series can be summed, leading to a compact analytical expression for the $O(1 / N)$ correction to the KM law. The correctness of this analytical formula is confirmed by its very good agreement with numerical diagonalization results. In addition, our approach allows us to identify the largest eigenvalue, separated from the continuous band by a gap, as a singularity in the $O(1 / N)$ correction to the resolvent. To our knowledge, a closely related problem has been considered so far only in some recent works [18 20], where it is shown rigorously that the fluctuations of the linear eigenvalue functional of RRGs converge to a random variable defined in terms of a sum over cyclically non-backtracking walks of all possible lengths.

The rest of the paper is organized as follows. In the next section we define the ensemble of RRGs. In section 3 we explain how to recast the problem in terms of a saddle- 
point integral using the replica method, and how one can integrate the fluctuations around the saddle-point solution. In section 4 the loop series for the $O(1 / N)$ correction to the eigenvalue distribution is obtained in replica symmetry, while the isolated eigenvalue and the final analytical expression for the finite size correction to the continuous band, together with a comparison with direct diagonalization results, are presented in section 5. In the last section we present some final remarks. The appendix A shows more details on how to derive the saddle-point integral with the replica method, while the appendix B discusses the correspondence between our results and those of reference [20].

\section{THE ENSEMBLE OF RANDOM REGULAR GRAPHS}

Let us consider the adjacency matrix $\boldsymbol{A}$ of an undirected random graph containing $N$ nodes or vertices, without self-loops and multiple edges between adjacent nodes [1]. The $N \times N$ symmetric random matrix $\boldsymbol{A}$ specifies the topology of the graph and it is constructed by setting $A_{i j}=1$ if there is an edge between nodes $i$ and $j$, and $A_{i j}=0$ otherwise. Defining the eigenvalues of $\boldsymbol{A}$ as $\lambda_{1}, \ldots, \lambda_{N}$, the average spectral density reads

$$
\rho^{(N)}(\lambda)=\left\langle\frac{1}{N} \sum_{\alpha=1}^{N} \delta\left(\lambda-\lambda_{\alpha}\right)\right\rangle,
$$

with $\langle\ldots\rangle$ denoting the ensemble average over the distribution of $\boldsymbol{A}$. We study an ensemble of random $c$-regular graphs, where each node is connected to $c \geq 3$ neighbors and the adjacency matrix is drawn from the distribution

$$
\begin{aligned}
p\left(\left\{A_{i<j}\right\}\right) & =\frac{1}{\mathcal{A}_{N}}\left[\prod_{i<j}\left(\frac{c}{N} \delta_{A_{i j}, 1}+\left(1-\frac{c}{N}\right) \delta_{A_{i j}, 0}\right)\right] \\
& \times\left[\prod_{i=1}^{N} \delta_{c, \sum_{j=1}^{N} A_{i j}}\right], \quad A_{i i}=0
\end{aligned}
$$

The product $\prod_{i<j}$ runs over all distinct pairs of nodes and $\mathcal{A}_{N}$ is the normalization factor. In this model, the probability that two nodes are connected by an edge is $c / N$, and the Kronecker $\delta$ ensures that all vertices are adjacent to $c$ neighbors.

The averaged resolvent associated to $\boldsymbol{A}$ can be defined as

$$
R^{(N)}(z)=\frac{1}{N}\langle\operatorname{Tr} \boldsymbol{G}(z)\rangle
$$

where the matrix $\boldsymbol{G}(z)$ is given by $\boldsymbol{G}(z)=(z-\boldsymbol{A})^{-1}$ and $z=\lambda-i \eta$ contains the regularizer $\eta>0$. The resolvent $R^{(N)}(z)$ is an analytic function in the lower half sector of the complex plane, except at the points or segments of the real axis corresponding to the eigenvalues of $\boldsymbol{A}$, at which $R^{(N)}(z)$ exhibits singularities. In general, the poles of $R^{(N)}(z)$ can be different than the simple poles exhibited by $\operatorname{Tr} \boldsymbol{G}(z)$, since the latter quantity is the resolvent before the average over the distribution of $\boldsymbol{A}$ is performed. The average distribution of eigenvalues is extracted from the limiting procedure

$$
\rho^{(N)}(\lambda)=\frac{1}{\pi} \lim _{\eta \rightarrow 0^{+}} \operatorname{Im}\left[R^{(N)}(z)\right] .
$$

By introducing the generating function

$$
\begin{aligned}
Z_{N}(z) & =\int\left(\prod_{i=1}^{N} d \phi_{i}\right) \exp \left(-\frac{i z}{2} \sum_{i=1}^{N} \phi_{i}^{2}\right) \\
& \times \exp \left(\frac{i}{2} \sum_{i j=1}^{N} \phi_{i} A_{i j} \phi_{j}\right)
\end{aligned}
$$

$R^{(N)}(z)$ is rewritten as follows

$$
R^{(N)}(z)=-\frac{2}{N} \frac{\partial}{\partial z}\left\langle\ln Z_{N}(z)\right\rangle
$$

In this way, we formulate the problem of computing $\rho^{(N)}(\lambda)$ in the language of statistical mechanics of disordered systems. According to eqs. (446), in order to calculate $\rho^{(N)}(\lambda)$ and its finite size fluctuations, one needs to study the average energy density of a system with real valued "spins" $\phi_{1}, \ldots, \phi_{N}$ placed on the vertices of a random regular graph and interacting through ferromagnetic couplings.

With the purpose of computing the average of the "free-energy" $\ln Z_{N}(z)$ over the random graph topology, we invoke the replica method [14, 15, 21, 22]

$$
R^{(N)}(z)=-2 \frac{\partial}{\partial z} \lim _{n \rightarrow 0} \frac{\partial}{\partial n} \frac{1}{N} \ln \left\langle\left[Z_{N}(z)\right]^{n}\right\rangle .
$$

The idea consists in calculating the average $\langle\ldots\rangle$ of integer powers of the generating function and, once the limit $N \rightarrow \infty$ is performed, the number of replicas is analytically continued to $n \rightarrow 0$. In this setting, the computation of $\left\langle\left[Z_{N}(z)\right]^{n}\right\rangle$ is written in terms of an integral over an order-parameter functional which can be solved, in the limit $N \rightarrow \infty$, by means of the saddlepoint method, leading to the KM distribution. As we will discuss in the next section, the $O(1 / N)$ correction to $\lim _{N \rightarrow \infty} \rho^{(N)}(\lambda)$ arises from the fluctuations of the order-parameter around the saddle-point solution. 


\section{THE SADDLE-POINT INTEGRAL AND THE FLUCTUATIONS AROUND THE STATIONARY SOLUTION}

The average of the replicated generating function is given by

$$
\begin{aligned}
\left\langle\left[Z_{N}(z)\right]^{n}\right\rangle & =\int\left(\prod_{i=1}^{N} d \boldsymbol{\phi}_{i}\right) \exp \left[-\frac{i z}{2} \sum_{i=1}^{N} \boldsymbol{\phi}_{i}^{2}\right] \\
& \times\left\langle\exp \left(i \sum_{i<j} A_{i j} \boldsymbol{\phi}_{i} \cdot \boldsymbol{\phi}_{j}\right)\right\rangle
\end{aligned}
$$

with $\phi=\left(\phi^{1}, \ldots, \phi^{n}\right)$ denoting a vector in the $n$ dimensional replica space. The average over the distribution $p\left(\left\{A_{i<j}\right\}\right)$ is calculated using integral representations for the Kronecker $\delta$ 's in eq. (2). After expanding the integrand exponent in eq. (8) up to order $O\left(N^{0}\right)$, site decoupling is achieved through the introduction of appropriate order-parameters, which leads to the compact expression (see the appendix A)

$$
\left\langle\left[Z_{N}(z)\right]^{n}\right\rangle=\sqrt{\operatorname{det}(c \boldsymbol{U})} \int \mathcal{D} \Psi \exp \left(-N S^{(N)}[\Psi]\right) .
$$

The object $\Psi(\phi)$ is the functional order-parameter and $\boldsymbol{U}$ can be seen as a matrix in the configuration space of the replica vectors, with elements $U(\boldsymbol{\phi}, \boldsymbol{\psi})=\exp (i \boldsymbol{\phi} . \boldsymbol{\psi})$. The functional integration measure can be intuitively written as $\mathcal{D} \Psi=\prod_{\{\phi\}} \sqrt{N / 2 \pi} d \Psi(\phi)$, where the product runs over all possible values of the vector $\phi$. The action $S^{(N)}[\Psi]$ has been expanded up to order $O\left(N^{-1}\right)$

$$
S^{(N)}[\Psi]=S_{0}[\Psi]+\frac{1}{N} S_{1}[\Psi]
$$

where the coefficients are given by

$$
\begin{aligned}
S_{0}[\Psi] & =\frac{c}{2} \int d \boldsymbol{\phi} d \boldsymbol{\psi} \Psi(\boldsymbol{\phi}) U(\boldsymbol{\phi}, \boldsymbol{\psi}) \Psi(\boldsymbol{\psi})-\frac{c}{2} \\
& -\ln \left[\int d \boldsymbol{\phi} H_{z}(\boldsymbol{\phi})\left(\int d \boldsymbol{\psi} U(\boldsymbol{\phi}, \boldsymbol{\psi}) \Psi(\boldsymbol{\psi})\right)^{c}\right], \\
S_{1}[\Psi] & =\frac{1}{4}\left(c^{2}+1\right)+\frac{(c-1)}{2} \int d \boldsymbol{\phi} r(\boldsymbol{\phi}) U(\boldsymbol{\phi}, \boldsymbol{\phi}) \\
& +\frac{(c-1)^{2}}{4} \int d \boldsymbol{\phi} d \boldsymbol{\psi} r(\boldsymbol{\phi})[U(\boldsymbol{\phi}, \boldsymbol{\psi})]^{2} r(\boldsymbol{\psi}) \\
& -\frac{c^{2}}{2} \int d \boldsymbol{\phi} d \boldsymbol{\psi} \Psi(\boldsymbol{\phi}) U(\boldsymbol{\phi}, \boldsymbol{\psi}) \Psi(\boldsymbol{\psi})-\frac{1}{2} \ln 2 .
\end{aligned}
$$

In the above expressions we have defined

$$
\begin{aligned}
H_{z}(\boldsymbol{\phi}) & =\exp \left(-\frac{i z}{2} \boldsymbol{\phi}^{2}\right) \\
r(\boldsymbol{\phi}) & =\frac{H_{z}(\boldsymbol{\phi})\left(\int d \boldsymbol{\psi} U(\boldsymbol{\phi}, \boldsymbol{\psi}) \Psi(\boldsymbol{\psi})\right)^{c-2}}{\int d \boldsymbol{\phi} H_{z}(\boldsymbol{\phi})\left(\int d \boldsymbol{\psi} U(\boldsymbol{\phi}, \boldsymbol{\psi}) \Psi(\boldsymbol{\psi})\right)^{c}}
\end{aligned}
$$

The details involved in the derivation of eqs. (9, 14 are discussed in the appendix $\mathrm{A}$.

In the limit $N \rightarrow \infty$, the integral in eq. (9) is dominated by the stationary solution $\Psi_{s}(\phi)$ fulfilling

$$
\left.\frac{\delta S_{0}[\Psi]}{\delta \Psi(\phi)}\right|_{\Psi_{s}}=0
$$

from which follows the saddle-point equation

$$
\begin{aligned}
\Psi_{s}(\boldsymbol{\phi}) & =\frac{H_{z}(\boldsymbol{\phi})\left(\int d \boldsymbol{\psi} U(\boldsymbol{\phi}, \boldsymbol{\psi}) \Psi_{s}(\boldsymbol{\psi})\right)^{c-1}}{\int d \boldsymbol{\phi} H_{z}(\boldsymbol{\phi})\left(\int d \boldsymbol{\psi} U(\boldsymbol{\phi}, \boldsymbol{\psi}) \Psi_{s}(\boldsymbol{\psi})\right)^{c}} \\
& =r_{s}(\boldsymbol{\phi}) \int d \boldsymbol{\psi} U(\boldsymbol{\phi}, \boldsymbol{\psi}) \Psi_{s}(\boldsymbol{\psi})
\end{aligned}
$$

In order to extract the $O(1 / N)$ correction to the distribution of eigenvalues we need to consider the effect of finite size fluctuations in $\Psi_{s}(\phi)$. The full action $S^{(N)}[\Psi]$ can be formally expanded around $\Psi_{s}(\phi)$ as follows

$$
\begin{aligned}
S^{(N)}[\Psi] & =S^{(N)}\left[\Psi_{s}\right]+\left.\int d \phi \frac{\delta S^{(N)}[\Psi]}{\delta \Psi(\phi)}\right|_{\Psi_{s}}\left[\Psi(\phi)-\Psi_{s}(\phi)\right] \\
& +\left.\frac{1}{2} \int d \boldsymbol{\phi} d \boldsymbol{\phi} \frac{\delta^{2} S^{(N)}[\Psi]}{\delta \Psi(\phi) \delta \Psi(\boldsymbol{\psi})}\right|_{\Psi_{s}} \\
& \times\left[\Psi(\boldsymbol{\phi})-\Psi_{s}(\boldsymbol{\phi})\right]\left[\Psi(\boldsymbol{\psi})-\Psi_{s}(\boldsymbol{\psi})\right]
\end{aligned}
$$

Assuming that the deviations from $\Psi_{s}(\phi)$ are of $O(1 / \sqrt{N})$ and retaining terms up to order $O(1 / N)$ in the above expansion, we substitute eq. (17) in eq. (9) and integrate over the Gaussian fluctuations to obtain

$$
\left\langle\left[Z_{N}(z)\right]^{n}\right\rangle=\frac{\sqrt{\operatorname{det}(c \boldsymbol{U})}}{\sqrt{\operatorname{det} \boldsymbol{J}_{0}}} \exp \left(-N S_{0}\left[\Psi_{s}\right]-S_{1}\left[\Psi_{s}\right]\right),
$$

where eq. (10) has been used. The elements of $\boldsymbol{J}_{0}$ read

$$
J_{0}(\boldsymbol{\phi}, \boldsymbol{\psi})=\left.\frac{\delta^{2} S_{0}[\Psi]}{\delta \Psi(\phi) \delta \Psi(\boldsymbol{\psi})}\right|_{\Psi_{s}}
$$

The explicit computation of the derivatives in eq. (19) and the subsequent use of eq. (16) leads to the following expression for $\boldsymbol{J}_{0}$

$$
\boldsymbol{J}_{0}=c \boldsymbol{U}-c \boldsymbol{U} \boldsymbol{T}
$$

where we have introduced the matrices

$$
T(\boldsymbol{\phi}, \boldsymbol{\psi})=(c-1) M(\boldsymbol{\phi}, \boldsymbol{\psi})-c \int d \boldsymbol{\psi}^{\prime} U\left(\boldsymbol{\psi}, \boldsymbol{\psi}^{\prime}\right) \Psi_{s}(\boldsymbol{\phi}) \Psi_{s}\left(\boldsymbol{\psi}^{\prime}\right)
$$

and

$$
M(\boldsymbol{\phi}, \boldsymbol{\psi})=U(\boldsymbol{\phi}, \boldsymbol{\psi}) r_{s}(\boldsymbol{\phi})
$$


By inserting eq. (20) in eq. (18) and employing the identity $\ln \operatorname{det} \boldsymbol{X}=\operatorname{Tr} \ln \boldsymbol{X}$ (here $\boldsymbol{X}$ denotes a generic matrix), we obtain the expression

$$
\frac{1}{N} \ln \left\langle\left[Z_{N}(z)\right]^{n}\right\rangle=-S_{0}\left[\Psi_{s}\right]-\frac{1}{N} S_{1}\left[\Psi_{s}\right]+\frac{1}{N} \sum_{L=1}^{\infty} \frac{\operatorname{Tr} \boldsymbol{T}^{L}}{2 L} .
$$

By substituting eq. (23) in eq. (7) and noting that the following identity holds

$$
\operatorname{Tr} \boldsymbol{T}^{L}=(-1)^{L}+(c-1)^{L}\left(\operatorname{Tr} \boldsymbol{M}^{L}-1\right),
$$

the first two terms of the series in eq. (23) cancel exactly with $S_{1}\left[\Psi_{s}\right]$ and we arrive at the following expression for $R^{(N)}(z)$

$$
R^{(N)}(z)=R_{0}(z)+\frac{1}{N} R_{1}(z),
$$

where

$$
\begin{aligned}
& R_{0}(z)=2 \frac{\partial}{\partial z} \lim _{n \rightarrow 0} \frac{\partial}{\partial n} S_{0}\left[\Psi_{s}\right], \\
& R_{1}(z)=2 \frac{\partial}{\partial z} \lim _{n \rightarrow 0} \frac{\partial}{\partial n} \sum_{L=3}^{\infty} \frac{(c-1)^{L}}{2 L}\left(1-\operatorname{Tr} \boldsymbol{M}^{L}\right) .
\end{aligned}
$$

This formula should be compared to similar formulae in [16, 17]. Substituting eq. (25) in eq. (4), we obtain the leading term $\rho_{0}(\lambda)$ and the $O(1 / N)$ correction $\rho_{1}(\lambda)$ to the eigenvalue distribution:

$\rho_{0}(\lambda)=\frac{1}{\pi} \lim _{\eta \rightarrow 0^{+}} \operatorname{Im}\left[R_{0}(z)\right], \quad \rho_{1}(\lambda)=\frac{1}{\pi} \lim _{\eta \rightarrow 0^{+}} \operatorname{Im}\left[R_{1}(z)\right]$.

In the next section we show how the limit $n \rightarrow 0$ is taken by assuming a particular form for the saddle-point solution $\Psi_{s}(\phi)$.

\section{THE DISTRIBUTION OF EIGENVALUES IN THE REPLICA SYMMETRIC THEORY}

The structure of eq. (16) suggests that we seek for a saddle-point solution $\Psi_{s}(\phi)$ invariant under orthogonal transformations. Indeed, it has been established that the replica symmetric (RS) saddle-point, which preserves both rotational and permutation symmetry in the replica space, yields exact results for the eigenvalue distribution of several sparse random graph models [15, 2126]. In particular, the correct analytical expression for $\lim _{N \rightarrow \infty} \rho^{(N)}(\lambda)$ in the case of regular random graphs is recovered by the RS solution. These results are also confirmed by reference [27], where the exactness of the RS assumption is proved rigorously for a large class of sparse random graphs with arbitrary degree distributions.

We thus assume that $\Psi_{s}(\phi)$ is an uncountable superposition of Gaussians 21, 22]

$$
\Psi_{s}(\phi)=\frac{1}{\mathcal{F}(n)} \int d g Q(g) \prod_{\alpha=1}^{n}\left(\frac{i}{2 \pi g}\right)^{\frac{1}{2}} \exp \left(\frac{-i \phi_{\alpha}^{2}}{2 g}\right),
$$

where $Q(g)$ is the normalized distribution of the complex variance $g$ with $\operatorname{Im} g>0$, such that the above integral is convergent. The factor $\mathcal{F}(n)$ accounts for the fact that $\Psi_{s}(\phi)$ is not normalized for arbitrary $n$, as can be noted from eq. (16). Plugging eq. (29) into eq. (16) and integrating over $\phi$, one can determine $\mathcal{F}(n)$ up to order $O(n)$

$$
\begin{aligned}
{[\mathcal{F}(n)]^{2} } & =1+\frac{n}{2} \int d g W(g) \ln \left(\frac{2 \pi g}{i}\right) \\
& -\frac{n}{2} \int d g Q(g) \ln \left(\frac{2 \pi g}{i}\right),
\end{aligned}
$$

and, in addition, the self-consistent equations for the distributions $Q(g)$ and $W(g)$

$$
\begin{aligned}
& Q(g)=\int\left(\prod_{k=1}^{c-1} d g_{k} Q\left(g_{k}\right)\right) \delta\left(g-\frac{1}{z-\sum_{k=1}^{c-1} g_{k}}\right), \\
& W(g)=\int\left(\prod_{k=1}^{c} d g_{k} Q\left(g_{k}\right)\right) \delta\left(g-\frac{1}{z-\sum_{k=1}^{c} g_{k}}\right) .
\end{aligned}
$$

Equations (31) and (32) can be also derived trough the more intuitive cavity method, where a clear physical interpretation emerges [28]. The function $W(g)$ is the distribution of $\left\{\boldsymbol{G}_{i i}(z)\right\}_{i=1, \ldots, N}$, while $Q(g)$ is the distribution of the diagonal elements of $\boldsymbol{G}(z)$ on the cavity graph, namely, a graph where a randomly chosen vertex and all its edges are removed. It is straightforward to check that $Q(g)=\delta\left(g-g_{c}\right)$ and $W(g)=\delta\left[g-\left(z-c g_{c}\right)^{-1}\right]$ solve, respectively, eqs. (31) and (32), with $g_{c}$ denoting one of the roots of the quadratic equation

$$
(c-1) g_{c}^{2}-z g_{c}+1=0 .
$$

The fact that $Q(g)$ and $W(g)$ are delta peak distributions simply reflects the absence of fluctuations on the degrees and on the edges of the graph.

One needs to be careful in choosing the root of eq. (33) depending on the value of $z$. The natural choice for $g_{c}$ is the following

$$
g_{c}=\left\{\begin{array}{ll}
\frac{1}{2(c-1)}\left(z+\sqrt{z^{2}-\lambda_{b}^{2}}\right) & \text { if }|z|<\left|\lambda_{b}\right| \\
\frac{1}{2(c-1)}\left(z-\sqrt{z^{2}-\lambda_{b}^{2}}\right) & \text { if }|z| \geq\left|\lambda_{b}\right|
\end{array},\right.
$$

where $\left|\lambda_{b}\right|=2 \sqrt{c-1}$. Equation (34) ensures that the leading term of the resolvent $R_{0}(z)$ is an analytic function of $z=\lambda-i \eta$. Besides that, this choice for $g_{c}$ reproduces the correct physical behavior $R_{0}(z)=1 / z$ for $|z| \rightarrow \infty$, since $g_{c} \rightarrow 0$ in this case [40]. This decay of $R_{0}(z)$ implies in the normalization $\int d \lambda \rho_{0}(\lambda)=1$, as can be noted from the Stieltjes transform of $\rho^{(N)}(\lambda)$.

Inserting the RS ansatz for $\Psi_{s}(\phi)$ in eq. (11) and taking the limit $n \rightarrow 0$, an analytical expression for $R_{0}(z)$ is derived through eq. (26). For $\eta \rightarrow 0^{+}, R_{0}(z)$ has a 
nonzero imaginary part only if $|\lambda|<\left|\lambda_{b}\right|$, from which the KM law follows using eq. (28)

$$
\rho_{0}(\lambda)=\left\{\begin{array}{cc}
\frac{c}{2 \pi} \frac{\sqrt{\lambda_{b}^{2}-\lambda^{2}}}{\left(c^{2}-\lambda^{2}\right)} & \text { for }|\lambda|<\left|\lambda_{b}\right| \\
0 & \text { for }|\lambda| \geq\left|\lambda_{b}\right|
\end{array} .\right.
$$

For the calculation of $R_{1}(z)$ one needs to obtain the RS form of $r_{s}(\phi)$. This is achieved by substituting eq. (29) in eq. (14) and expanding the result up to order $O(n)$

$$
r_{s}(\phi)=\left[1-\frac{n}{2} \ln \left(\frac{2 \pi g_{c}}{i}\right)\right] \exp \left[\frac{i \phi^{2}}{2}\left((c-2) g_{c}-z\right)\right]
$$

which allows us to perform the limit $n \rightarrow 0$ in eq. (27) and derive the expression:

$$
R_{1}(z)=\sum_{L=3}^{\infty} \frac{(c-1)^{L}}{2 L} \frac{\partial}{\partial z}\left(L \ln g_{c}-2 \ln Z_{L}^{(c)}\left(g_{c}\right)\right)
$$

The object $Z_{L}^{(c)}\left(g_{c}\right)$, defined analogously to eq. (5), is the generating function associated to the $L \times L$ tridiagonal matrix $\mathcal{H}$, whose elements are given by

$$
\mathcal{H}_{i j}=(c-2) g_{c} \delta_{i j}+\delta_{i, j-1}+\delta_{i, j+1}, \quad i+N \equiv i
$$

The physical meaning of eq. (37) is quite transparent. The object $\frac{\partial}{\partial z} \ln Z_{L}^{(c)}\left(g_{c}\right)$ can be seen as the resolvent of a $1 \mathrm{D}$ closed chain or loop of length $L$, where each node receives an effective field $g_{c}$ from each one of its $(c-2)$ neighbors living outside the loop. We point out that, at the level of the $O(1 / N)$ correction, each node belongs only to a single loop, i.e., there are no intersecting loops, since these objects arise on average in a fraction $O\left(1 / N^{2}\right)$ of nodes. The quantity $\frac{\partial}{\partial z} \ln g_{c}$ is the resolvent of a $1 \mathrm{D}$ closed chain of infinite length 29]. As a consequence, the $O(1 / N)$ fluctuations due to all loops of a certain length $L$ modify $R^{(N)}(z)$ by a term proportional on average to the difference between the resolvent of an infinite loop and the resolvent of a finite loop of length $L$. The weight $\frac{(c-1)^{L}}{2 L}$ is the average number of loops of length $L$ in a regular random graph of degree $c[30,31]$. A result analogous to eq. (37) has been derived in the study of the $O(1 / N)$ corrections to the free-energy of disordered spin systems defined on sparse random graphs [16].

The Gaussian integral in $Z_{L}^{(c)}\left(g_{c}\right)$ is evaluated using the eigenvalues of the matrix $\mathcal{H}$, given by $a_{n}=g_{c}(c-$ $2)+2 \cos (2 \pi n / L), n=0, \ldots, L-1$, which allows us to compute in eq. (37) the derivative with respect to $z$

$$
\begin{aligned}
R_{1}(z) & =\sum_{L=3}^{\infty} \frac{(c-1)^{L}}{2 L} \\
& \times\left[\sum_{n=0}^{L-1} \frac{\left(1-(c-2) \frac{\partial g_{c}}{\partial z}\right)}{\left(z-(c-2) g_{c}-2 \cos \left(\frac{2 \pi n}{L}\right)\right)}+\frac{L}{g_{c}} \frac{\partial g_{c}}{\partial z}\right] .
\end{aligned}
$$

From now on, the calculation depends, according to eq. (34), whether $|z|<\left|\lambda_{b}\right|$ or $|z| \geq\left|\lambda_{b}\right|$, from which the following expression for $\frac{\partial g_{c}}{\partial z}$ is obtained

$$
\frac{\partial g_{c}}{\partial z}=\left\{\begin{array}{cl}
\frac{g_{c}}{\sqrt{z^{2}-\lambda_{b}^{2}}} & \text { if }|z|<\left|\lambda_{b}\right| \\
-\frac{g_{c}}{\sqrt{z^{2}-\lambda_{b}^{2}}} & \text { if }|z| \geq\left|\lambda_{b}\right|
\end{array}\right.
$$

This leads to the following simplified form of $R_{1}(z)$

$$
\begin{aligned}
R_{1}(z) & =\frac{\operatorname{sign}\left(|z|-\left|\lambda_{b}\right|\right)}{2 \sqrt{z^{2}-\lambda_{b}^{2}}} \sum_{L=3}^{\infty}(c-1)^{L} \\
& \times\left\{\frac{[(c-2) z+c B(z)]}{2 \pi} \mathcal{G}_{L}(z)-1\right\},
\end{aligned}
$$

where we have defined

$$
\begin{aligned}
\mathcal{G}_{L}(z) & =\frac{2 \pi}{L} \sum_{n=0}^{L-1} F_{z}\left(x_{n}\right), \quad x_{n}=\frac{2 \pi n}{L}, \\
B(z) & =\operatorname{sign}\left(|z|-\left|\lambda_{b}\right|\right) \sqrt{z^{2}-\lambda_{b}^{2}}
\end{aligned}
$$

with

$$
F_{z}(x)=\frac{1}{c z+(c-2) B(z)-4(c-1) \cos (x)}
$$

and $\operatorname{sign}(0) \equiv 1$. In the limit $L \rightarrow \infty, \mathcal{G}_{L}(z)$ becomes simply an integral of the periodic function $F_{z}(x)$, which is solved using standard contour integration methods. The result reads

$$
\lim _{L \rightarrow \infty} \mathcal{G}_{L}(z)=\int_{0}^{2 \pi} d x F_{z}(x)=\frac{2 \pi}{(c-2) z+c B(z)} .
$$

It follows that the individual terms of the loop series in eq. (41) are composed of the exponential growing factor $(c-1)^{L}$ multiplied by a function that is going to zero for $L \rightarrow \infty$. The key point consists in understanding how fast this function vanishes as a function of $L$. We will see in the next section that one can extract the explicit dependence of the summands with respect to $L$ by borrowing techniques used to compute the discretization error in the trapezoidal method of numerical integration.

\section{THE LOOP SERIES AND THE FINAL EXPRESSION FOR $\rho_{1}(\lambda)$}

The problem of studying how $\mathcal{G}_{L}(z)$ approaches its asymptotic form $\lim _{L \rightarrow \infty} \mathcal{G}_{L}(z)$ is equivalent to evaluate the error of replacing the sum in eq. (42) by the integral of eq. (45). This is analogous to compute the discretization error in some numerical integration methods, where several techniques are available [32]. Here we extract the dependence of $\mathcal{G}_{L}(z)$ with respect to $L$ via a Fourier analysis, following steps typically employed to compute the discretization error in the trapezoidal rule of numerical integration 32 34]. 
Let us expand $F_{z}(x)$ in a Fourier series

$$
\begin{aligned}
F_{z}(x) & =\frac{a_{0}}{2}+\sum_{k=1}^{\infty} a_{k} \cos (k x), \\
a_{k} & =\frac{1}{\pi} \int_{-\pi}^{\pi} d x \cos (k x) F_{z}(x),
\end{aligned}
$$

and assume that this series converges at the points $x_{n}$ $(n=0, \ldots, L-1)$ defined in eq. (42). Plugging the above expansion into $\mathcal{G}_{L}(z)$ and noting that $\lim _{L \rightarrow \infty} \mathcal{G}_{L}(z)=$ $\pi a_{0}$, we obtain an exact equation for the deviation of $\mathcal{G}_{L}(z)$ with respect to its $L \rightarrow \infty$ limit

$$
\mathcal{G}_{L}(z)-\lim _{L \rightarrow \infty} \mathcal{G}_{L}(z)=2 \sum_{m=1}^{\infty} \int_{-\pi}^{\pi} d x \cos (m L x) F_{z}(x) .
$$

The asymptotic behavior of $\mathcal{G}_{L}(z)$ is governed by the convergence rate of the Fourier series for $F_{z}(x)$, in full analogy with the error formula for the trapezoidal quadrature 33, 34. In order to make further progress, eq. (48) is substituted in eq. (41) and the above integral over $F_{z}(x)$ is transformed in a contour integral along the unit circle in the complex plane, traversed once in the counterclockwise direction

$$
\begin{aligned}
R_{1}(z) & =\frac{-\left[\operatorname{sign}\left(|z|-\left|\lambda_{b}\right|\right)(c-2) z+c \sqrt{z^{2}-\lambda_{b}^{2}}\right]}{4 \pi i(c-1) \sqrt{z^{2}-\lambda_{b}^{2}}} \\
& \times \sum_{L=3}^{\infty}(c-1)^{L} \sum_{m=1}^{\infty} \oint \frac{d \omega \omega^{m L}}{\omega^{2}+2 Z_{z} \omega+1},
\end{aligned}
$$

where

$$
Z_{z}=-\frac{1}{4(c-1)}[c z+(c-2) B(z)] .
$$

The rest of the analysis amounts to study, in the integrand of eq. (49), the behavior of the poles, i.e., the roots of the quadratic equation $\omega^{2}+2 Z_{z} \omega+1=0$. In general, one root $\omega_{d}$ lies inside the unit circle in the complex plane, while the other root $\omega_{f}$ lies outside. Using eq. (34) and the quadratic equation $g_{c}=\left[z-(c-1) g_{c}\right]^{-1}$, one can show that $Z_{z}=-\frac{1}{2}\left(g_{c}+g_{c}^{-1}\right)$, from which the roots $\omega_{d}$ and $\omega_{f}$ are computed explicitly [41]

$$
\omega_{d}=g_{c}, \quad \omega_{f}=\frac{1}{g_{c}} .
$$

This allows us to solve the contour integral in eq. (49) through the residue theorem and derive the following expression

$$
R_{1}(z)=\mathcal{C}(z) \sum_{L=3}^{\infty}(c-1)^{L} \frac{g_{c}^{L}}{1-g_{c}^{L}}
$$

where the prefactor $\mathcal{C}(z)$ is given by

$$
\mathcal{C}(z)=\left\{\begin{array}{cc}
\frac{\left(z-c g_{c}\right) g_{c}}{\sqrt{z^{2}-\lambda_{b}^{2}\left(g_{c}^{2}-1\right)}} & \text { if }|z|<\left|\lambda_{b}\right| \\
-\frac{\left(z-c g_{c}\right) g_{c}}{\sqrt{z^{2}-\lambda_{b}^{2}}\left(g_{c}^{2}-1\right)} & \text { if }|z| \geq\left|\lambda_{b}\right|
\end{array}\right.
$$

The $O(1 / N)$ correction $R_{1}(z)$ to the resolvent is an analytic function of $z$ with singularities located possibly only on the real axis. In the regime $|z| \rightarrow \infty$, we have that $g_{c}=O(1 / z)$ and $\mathcal{C}(z)=O(1 / z)$, such that the loop series in eq. (52) also converges to zero for large $z$. It follows that $R_{1}(z)$ vanishes faster than $1 / z$, which implies that $\int d \lambda \rho_{1}(\lambda)=0$, as can be checked using the Stieltjes transform of $\rho^{(N)}(\lambda)$. This is consistent with the normalization of both the full eigenvalue distribution $\rho^{(N)}(\lambda)$ and its leading term $\rho_{0}(\lambda)$. In the sequel we study, separately in the sectors $|z| \geq\left|\lambda_{b}\right|$ and $|z|<\left|\lambda_{b}\right|$, the behavior of $R_{1}(z)$ as $\eta \rightarrow 0^{+}$.

\section{A. $|\lambda| \geq\left|\lambda_{b}\right|$ : the isolated eigenvalue}

The idea now consists in setting $z=\lambda$ and then making an analytical continuation from $\lambda \rightarrow \infty$, where $R_{1}(\lambda)$ is convergent, to smaller values of $\lambda$. In the regime $|\lambda| \geq$ $\left|\lambda_{b}\right|$, the quantity $g_{c}$ reads

$$
g_{c}=\frac{1}{2(c-1)}\left(\lambda-\operatorname{sign}(\lambda) \sqrt{\lambda^{2}-\lambda_{b}^{2}}\right) .
$$

One can check that, for $\lambda>c, g_{c}$ fulfills $0<g_{c}<1 /(c-1)$ and the loop series in eq. (52) is convergent. For $\lambda=$ $c$, we have that $g_{c}=1 /(c-1)$, and the loop series in eq. (52) becomes divergent. This singular behavior is consistent with the existence of an isolated eigenvalue, located at $\lambda=c$, outside of the support $\left(-\left|\lambda_{b}\right|,\left|\lambda_{b}\right|\right)$ of the continuous part of the spectrum. Indeed, for this simple model of RRGs, this isolated eigenvalue can be computed directly from the eigenvalue equation and it corresponds to the uniform eigenvector.

For $\left|\lambda_{b}\right|<\lambda<c$, we have that $1 /(c-1)<g_{c}<$ $1 / \sqrt{c-1}$ and the loop series of eq. (52) is divergent. However, we can rewrite this series as follows

$$
\sum_{L=3}^{\infty}(c-1)^{L} \frac{g_{c}^{L}}{1-g_{c}^{L}}=\sum_{L=3}^{\infty}(c-1)^{L} g_{c}^{L}+\sum_{L=3}^{\infty}(c-1)^{L} \frac{g_{c}^{2 L}}{1-g_{c}^{L}}
$$

The second term on the right hand side is a convergent series, while we can assign a finite value for the summation of the first term using the standard expression for the geometric series 35], leading to a finite result for $R_{1}(\lambda)$ in the range $\left|\lambda_{b}\right|<\lambda<c$. Finally, we have that $g_{c}=1 / \sqrt{c-1}$ for $\lambda=\left|\lambda_{b}\right|$ and a second singularity arises, which corresponds to the edge of the continuous band.

For a given point $\lambda$ in the regime $\lambda<-\left|\lambda_{b}\right|, g_{c}$ is given by minus its value at $|\lambda|$. Thus the qualitative behavior of $R_{1}(\lambda)$ for $\lambda<-\left|\lambda_{b}\right|$ is completely analogous to the case $\lambda>\left|\lambda_{b}\right|$, with the exception that $R_{1}(\lambda)$ is finite for $\lambda=-c$, since the first term on the right hand side of eq. (55) is an alternating divergent series that can be summed using the summation formula for the geometric series 35]. Consequently, $R_{1}(\lambda)$ remains finite in the whole sector $\lambda<-\left|\lambda_{b}\right|$, exhibiting a singularity only at 
$\lambda=-\left|\lambda_{b}\right|$. We point out that, according to eq. (54), $g_{c} \in \mathbb{R}$ for $|\lambda| \geq\left|\lambda_{b}\right|$. This implies that, for the different sectors of $\lambda$ where $R_{1}(\lambda)$ attains a finite value, we have that $\rho_{1}(\lambda)=0$, since $R_{1}(\lambda)$ is also a real-valued function (see eqs. (52) and (53).

\section{B. $|\lambda|<\left|\lambda_{b}\right|$ : the continuous band of eigenvalues}

For $\eta \rightarrow 0^{+}$and $|\lambda|<\left|\lambda_{b}\right|, g_{c}$ is obtained from eq. (34)

$$
g_{c}=\frac{1}{2(c-1)}\left(\lambda+i \sqrt{\lambda_{b}^{2}-\lambda^{2}}\right) .
$$

Inserting the above form of $g_{c}$ in eq. (53), one can show that $\operatorname{Re} \mathcal{C}(\lambda)=0$. Thus, by taking the imaginary part of eq. (52), the following expression is derived for the $O(1 / N)$ correction $\rho_{1}(\lambda)$ to the continuous part of the eigenvalue distribution

$$
\rho_{1}(\lambda)=C(\lambda) \operatorname{Re}\left[\sum_{L=3}^{\infty}(c-1)^{L} \frac{g_{c}^{L}}{1-g_{c}^{L}}\right],
$$

with

$$
C(\lambda)=\frac{1}{\pi \sqrt{\lambda_{b}^{2}-\lambda^{2}}} .
$$

Equation (57) can be derived from the average of the finite size fluctuations of the linear eigenvalue functional defined in reference [20]. The correspondence between $\rho_{1}(\lambda)$ and the rigorous results of [20] is discussed in appendix $\mathrm{B}$

The last step consists in handling the loop series in eq. (57), which is irremediably divergent since $\left|g_{c}\right|=$ $1 / \sqrt{c-1}$. However, we can rewrite this series according to

$$
\begin{aligned}
\sum_{L=3}^{\infty}(c-1)^{L} \frac{g_{c}^{L}}{1-g_{c}^{L}} & =\sum_{L=3}^{\infty}(c-1)^{L} g_{c}^{L}+\sum_{L=3}^{\infty}(c-1)^{L} g_{c}^{2 L} \\
& +\sum_{L=3}^{\infty}(c-1)^{L} \frac{g_{c}^{3 L}}{\left(1-g_{c}^{L}\right)}
\end{aligned}
$$

and, despite the fact that the first two terms on the right hand side are divergent, they can be summed using the summation formula for the geometric series 35]. The series containing $g_{c}^{3 L}$ is clearly convergent and, in this way, we arrive at the final expression for $\rho_{1}(\lambda)$

$$
\begin{aligned}
\rho_{1}(\lambda) & =C(\lambda) \operatorname{Re}\left[\frac{(c-1) g_{c}}{1-(c-1) g_{c}}+\frac{(c-1) g_{c}^{2}}{1-(c-1) g_{c}^{2}}\right] \\
& +C(\lambda) \operatorname{Re}\left[\sum_{L=3}^{\infty}(c-1)^{L} \frac{g_{c}^{3 L}}{\left(1-g_{c}^{L}\right)}-K\left(g_{c}\right)\right],
\end{aligned}
$$

where the factor $K\left(g_{c}\right)$ accounts for the absence of the terms with $L=1$ and $L=2$ in eq (59):

$$
K\left(g_{c}\right)=(c-1) g_{c}+c(c-1) g_{c}^{2}+(c-1)^{2} g_{c}^{4} .
$$

Equation (60) constitutes the central result of this work: it provides the analytical expression for the $O(1 / N)$ correction to the KM distribution for $|\lambda|<\left|\lambda_{b}\right|$.

There is one important point as far as the behavior near $\pm\left|\lambda_{b}\right|$ is concerned. In the limit $\lambda \rightarrow \pm\left|\lambda_{b}\right|$, we have that $C(\lambda)$ diverges as $O\left(\left(\left|\lambda_{b}\right| \mp \lambda\right)^{-1 / 2}\right)$, while the real part of the loop series in eq. (60) is numerically shown to converge to a negative finite value. Thus $\rho_{1}(\lambda)$ is a distribution with integrable singularities at $\lambda= \pm\left|\lambda_{b}\right|$. There is also a contribution proportional to $\delta\left(\lambda \pm\left|\lambda_{b}\right|\right)$ because the resolvent has poles at these points. The details of the behavior at the band edges will not be investigated here.

In figure 1 we compare eq. (60) with direct diagonalization results of the adjacency matrix of regular random graphs with $N=500$, generated according to the algorithm presented in reference 36. The agreement between theoretical and numerical results is excellent. For finite $N$, the regular graph becomes sensibly non-bipartite due to the presence of loops, which is reflected in the breaking of the symmetry $\lambda \rightarrow-\lambda$ in $\rho_{1}(\lambda)$.

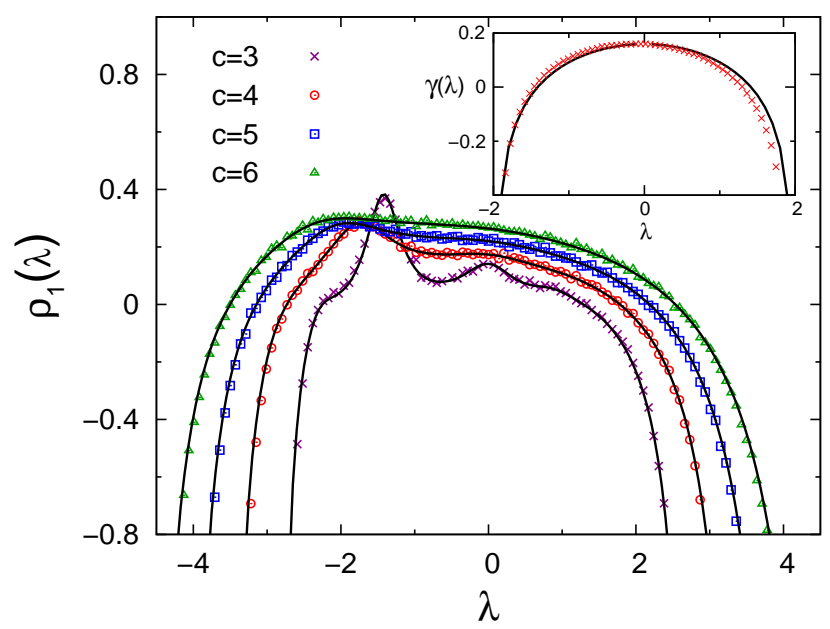

FIG. 1: The $O(1 / N)$ correction to the average eigenvalue distribution of the adjacency matrix of an ensemble of regular random graphs with degree $c$, where the isolated eigenvalue $\lambda=c$ has been omitted. In the main graph, the solid black curves depict the analytical result of eq. (60) for different $c$, while the symbols represent numerical diagonalization results obtained from matrices of size $N=500$. In the inset, the solid black line shows the analytical expression for $c \gg 1$, given by eq. (62), and the red symbols are direct diagonalization results for $c=40$ and $N=500$. The histograms from numerical diagonalizations are obtained by averaging the results over $5 \times 10^{6}$ samples.

After rescaling the adjacency matrix elements as $A_{i j} \rightarrow$ $\frac{A_{i j}}{\sqrt{c-1}}$, one can show that, in the regime $1 \ll c \ll N$, the dominant contribution to $\rho_{1}(\lambda)$ is given by $\rho_{1}(\lambda)=$ $c \gamma(\lambda)$, where the coefficient $\gamma(\lambda)$ reads

$$
\gamma(\lambda)=\frac{2-\lambda^{2}}{2 \pi \sqrt{4-\lambda^{2}}}
$$


The numerical diagonalization results converge for large $c$ to eq. (62), as illustrated in the inset of figure 1 Although the leading term $\rho_{0}(\lambda)$ converges to the Wigner semicircle law for $c \gg 1$, this is not the case for the $O(1 / N)$ fluctuations, as can be seen by comparing eq. (62) with the corresponding results in references [37, 38].

\section{FINAL REMARKS}

The average eigenvalue distribution of a regular random graph with $N$ vertices converges, in the limit $N \rightarrow$ $\infty$, to the well-known Kesten-McKay (KM) law. In this work we have derived an exact analytical expression for the $O(1 / N)$ correction to the KM law using the replica approach for random matrices. The $O(1 / N)$ correction is incorporated in the replica scheme by taking into account the $O(1 / \sqrt{N})$ fluctuations around the mean-field saddlepoint solution. Although the intermediate steps in the replica method are not very intuitive, the interpretation of the final expression for the $O(1 / N)$ fluctuations of the resolvent, cf. eq. (37), from which follows our analytical result, given by eq. (60), is rather clear: it consists of a sum over the average contributions coming from loops of all possible lengths, each loop of finite length contributing with a term proportional to the deviation of its effective resolvent with respect to the resolvent of an infinite loop. The approach discussed in this work is also capable to determine the isolated eigenvalue, since the latter has a weight of $O(1 / N)$ in the average eigenvalue distribution.

The ideas presented here can be possibly extended to more general random graph models including disordered edges and fluctuating connectivities, which opens the possibility to analyze, for instance, finite size fluctuations in the Anderson model on the Bethe lattice [3]. Despite the non-critical behavior of the average density of states along the localization transition, the study of finite size corrections in such mean-field models may provide some valuable insights on the influence of loops in the electronic properties of finite dimensional models. Besides that, the study of finite size corrections to the density of states can be considered as a warm up to the more complicated task of considering relevant quantities to the localization transition, such as the inverse participation ratio.

On the methodological side, a derivation of eq. (37) through the cavity method would be a meaningful exercise, since the latter approach, being conceptually simpler, usually provides additional physical insights, which are obscured by the replica calculation. Work along some of these lines is underway, following the lines of [16, 17].

Finally, it would be also interesting to examine the universality status of the level correlation function in the case of sparse random graph models [39], using the ideas presented in this paper.

\section{Acknowledgments}

GP thanks Gérard Ben Arous for fruitful discussions. FLM thanks Carlo Lucibello for interesting comments. The research leading to these results has received funding from the European Research Council (ERC) grant agreement No. 247328 (CriPheRaSy project), from the People Programme (Marie Curie Actions) of the European Union's Seventh Framework Programme FP7/2007-2013/ under REA grant agreement No. 290038 (NETADIS project) and from the Italian MIUR under the Basic Research Investigation Fund FIRB2008 program, grant No. RBFR08M3P4, and under the PRIN2010 program, grant code 2010HXAW77-008.

\section{Appendix A: Derivation of the saddle-point integral}

The purpose of this appendix is to discuss the main steps involved in the derivation of eq. (9). The average over the topological disorder in eq. (8) is calculated using integral representations for the Kronecker deltas in the distribution $p\left(\left\{A_{i<j}\right\}\right)$, leading to

$$
\begin{aligned}
\left\langle\left[Z_{N}(z)\right]^{n}\right\rangle & =\frac{1}{\mathcal{A}_{N}} \int\left(\prod_{i=1}^{N} d \phi_{i} H_{z}\left(\boldsymbol{\phi}_{i}\right)\right) \int_{0}^{2 \pi}\left(\prod_{i=1}^{N} \frac{d x_{i}}{2 \pi} e^{i c x_{i}}\right) \exp \left[\frac{1}{2} \sum_{i j=1}^{N} \ln \left[1+\frac{c}{N}\left(e^{-i\left(x_{i}+x_{j}\right)} U\left(\boldsymbol{\phi}_{i}, \boldsymbol{\phi}_{j}\right)-1\right)\right]\right] \\
& \times \exp \left[-\frac{1}{2} \sum_{i=1}^{N} \ln \left[1+\frac{c}{N}\left(e^{-2 i x_{i}} U\left(\boldsymbol{\phi}_{i}, \boldsymbol{\phi}_{i}\right)-1\right)\right]\right]
\end{aligned}
$$

where $U(\boldsymbol{\phi}, \boldsymbol{\psi})=\exp (i \phi . \psi)$ and $H_{z}(\boldsymbol{\phi})$ is defined by eq. (13). Since we are interested in the $O(1 / N)$ correction to the average spectrum, we need to determine the exponent of the above integrand up to $O\left(N^{0}\right)$. After performing an expansion in powers of $1 / N$, the sites are decoupled via the introduction, by means of the Fourier integral representation of the Dirac delta, of the functional order- 
parameters

$$
\begin{aligned}
& \mu_{1}(\boldsymbol{\phi})=\frac{1}{N} \sum_{i=1}^{N} \delta\left(\boldsymbol{\phi}-\boldsymbol{\phi}_{i}\right) e^{-i x_{i}}, \\
& \mu_{2}(\boldsymbol{\phi})=\frac{1}{N} \sum_{i=1}^{N} \delta\left(\boldsymbol{\phi}-\boldsymbol{\phi}_{i}\right) e^{-2 i x_{i}},
\end{aligned}
$$

which allows us to recast $\left\langle\left[Z_{N}(z)\right]^{n}\right\rangle$ in the form

$$
\begin{aligned}
& \left\langle\left[Z_{N}(z)\right]^{n}\right\rangle=\frac{\exp \left(-\frac{N c}{2}-\frac{c^{2}}{4}+\frac{c}{2}\right)}{\mathcal{A}_{N}} \int \mathcal{D} \mu_{1} \mathcal{D} \hat{\mu}_{1} \mathcal{D} \mu_{2} \mathcal{D} \hat{\mu}_{2} \\
& \times \exp \left[i \int d \boldsymbol{\phi}\left[\mu_{1}(\boldsymbol{\phi}) \hat{\mu}_{1}(\boldsymbol{\phi})+\mu_{2}(\boldsymbol{\phi}) \hat{\mu}_{2}(\boldsymbol{\phi})\right]\right] \\
& \times \exp \left[N \ln \mathcal{I}\left[\hat{\mu_{1}}, \hat{\mu_{2}}\right]-\frac{c}{2} \int d \boldsymbol{\phi} \mu_{2}(\boldsymbol{\phi}) U(\boldsymbol{\phi}, \boldsymbol{\phi})\right] \\
& \times \exp \left[\frac{c}{2}(N+c) \int d \boldsymbol{\phi} d \boldsymbol{\psi} \mu_{1}(\boldsymbol{\phi}) U(\boldsymbol{\phi}, \boldsymbol{\psi}) \mu_{1}(\boldsymbol{\psi})\right] \\
& \times \exp \left[-\frac{c^{2}}{4} \int d \boldsymbol{\phi} d \boldsymbol{\psi} \mu_{2}(\boldsymbol{\phi})(U(\boldsymbol{\phi}, \boldsymbol{\psi}))^{2} \mu_{2}(\boldsymbol{\psi})\right], \quad(\mathrm{A} 1)
\end{aligned}
$$

where

$$
\begin{aligned}
\mathcal{I}\left[\hat{\mu_{1}}, \hat{\mu_{2}}\right] & =\int d \phi H_{z}(\phi) \int_{0}^{2 \pi} \frac{d x}{2 \pi} \\
& \times \exp \left[i c x-\frac{i}{N} \hat{\mu_{1}}(\phi) e^{-i x}-\frac{i}{N} \hat{\mu_{2}}(\phi) e^{-2 i x}\right] .
\end{aligned}
$$

Each integration measure $\mathcal{D} \mu_{1}, \ldots, \mathcal{D} \hat{\mu}_{2}$ in eq. (A1) includes an unimportant factor $1 / \sqrt{2 \pi}$ coming from the Fourier representation of the Dirac delta function. The integral over $x$ in eq. (A2) is calculated using the powerseries representation

$$
\exp \left[-\frac{i}{N} \hat{\mu_{I}}(\phi) e^{-i I x}\right]=\sum_{k=0}^{\infty}\left(-\frac{i}{N} \hat{\mu_{I}}(\boldsymbol{\phi})\right)^{k} \frac{e^{-i I k x}}{k !},
$$

with $I=1,2$. By substituting eq. $\mathrm{A} 3$ in eq. $\mathrm{A2}$ and integrating over $x$, we obtain

$$
\mathcal{I}\left[\hat{\mu_{1}}, \hat{\mu_{2}}\right]=\int d \phi H_{z}(\phi) \sum_{k=0}^{\infty} \frac{\left[-i \hat{\mu_{2}}(\phi)\right]^{k}}{k !} \frac{\left[-i \hat{\mu_{1}}(\phi)\right]^{c-2 k}}{N^{c-k}(c-2 k) !} .
$$

After performing the rescaling $\hat{\mu}_{1}(\phi) \rightarrow N \hat{\mu}_{1}(\phi)$, eq. (A4) can be expanded up to $O(1 / N)$, which yields, after the substitution of the result in eq. (A1), the following expression

$$
\begin{aligned}
\left\langle\left[Z_{N}(z)\right]^{n}\right\rangle & =\frac{\exp \left(-\frac{N c}{2}-\frac{c^{2}}{4}+\frac{c}{2}\right)}{\mathcal{A}_{N}} \int \mathcal{D} \mu_{1} \mathcal{D} \hat{\mu}_{1} \mathcal{D} \mu_{2} \mathcal{D} \hat{\mu}_{2} \exp \left[i \int d \boldsymbol{\phi}\left(N \mu_{1}(\boldsymbol{\phi}) \hat{\mu}_{1}(\boldsymbol{\phi})+\mu_{2}(\boldsymbol{\phi}) \hat{\mu}_{2}(\boldsymbol{\phi})\right)\right] \\
& \times \exp \left[\frac{c}{2}(N+c) \int d \boldsymbol{\phi} d \boldsymbol{\psi} \mu_{1}(\boldsymbol{\phi}) U(\boldsymbol{\phi}, \boldsymbol{\psi}) \mu_{1}(\boldsymbol{\psi})-\frac{c}{2} \int d \boldsymbol{\phi} \mu_{2}(\boldsymbol{\phi}) U(\boldsymbol{\phi}, \boldsymbol{\phi})+N \ln \left(\int \frac{d \boldsymbol{\phi}}{c !} H_{z}(\boldsymbol{\phi})\left[-i \hat{\mu}_{1}(\boldsymbol{\phi})\right]^{c}\right)\right] \\
& \times \exp \left[-\frac{c^{2}}{4} \int d \boldsymbol{\phi} d \boldsymbol{\psi} \mu_{2}(\boldsymbol{\phi})(U(\boldsymbol{\phi}, \boldsymbol{\psi}))^{2} \mu_{2}(\boldsymbol{\psi})+i \int d \boldsymbol{\phi} \mathcal{R}\left[\hat{\mu}_{1}(\boldsymbol{\phi})\right] \hat{\mu}_{2}(\boldsymbol{\phi})\right]
\end{aligned}
$$

where we have defined

$$
\mathcal{R}\left[\hat{\mu}_{1}(\phi)\right]=c(c-1) \frac{H_{z}(\phi)\left[\hat{\mu}_{1}(\phi)\right]^{c-2}}{\int d \phi H_{z}(\phi)\left[\hat{\mu}_{1}(\phi)\right]^{c}},
$$

and $\mathcal{D} \hat{\mu}_{1}=\prod_{\{\phi\}} N / \sqrt{2 \pi} d \hat{\mu}_{1}(\phi)$, while the other integration measures are defined similarly, but without the factor $N$. Now one can integrate over $\hat{\mu}_{2}, \mu_{2}$ and $\mu_{1}$ to obtain

$$
\begin{aligned}
& \left\langle\left[Z_{N}(z)\right]^{n}\right\rangle=\frac{\exp \left(-\frac{N c}{2}-\frac{c^{2}}{4}+\frac{c}{2}\right)}{\mathcal{A}_{N}\left[\operatorname{det}\left(\boldsymbol{U}\left(-c-\frac{c^{2}}{N}\right)\right)\right]^{\frac{1}{2}}} \\
& \times \int \mathcal{D} \hat{\mu}_{1} \exp \left[\frac{c}{2} \int d \boldsymbol{\phi} \mathcal{R}\left[\hat{\mu}_{1}(\boldsymbol{\phi})\right] U(\boldsymbol{\phi}, \boldsymbol{\phi})\right] \\
& \times \exp \left[\frac{N}{2 c}\left(1-\frac{c}{N}\right) \int d \boldsymbol{\phi} d \boldsymbol{\psi} \hat{\mu}_{1}(\boldsymbol{\phi}) U^{-1}(\boldsymbol{\phi}, \boldsymbol{\psi}) \hat{\mu}_{1}(\boldsymbol{\psi})\right] \\
& \times \exp \left[-\frac{c^{2}}{4} \int d \boldsymbol{\phi} d \boldsymbol{\psi} \mathcal{R}\left[\hat{\mu}_{1}(\boldsymbol{\phi})\right](U(\boldsymbol{\phi}, \boldsymbol{\psi}))^{2} \mathcal{R}\left[\hat{\mu}_{1}(\boldsymbol{\psi})\right]\right] \\
& \times \exp \left[N \ln \left(\int \frac{d \boldsymbol{\phi}}{c !} H_{z}(\boldsymbol{\phi})\left[-i \hat{\mu}_{1}(\boldsymbol{\phi})\right]^{c}\right)\right],
\end{aligned}
$$


where the integration measure becomes $\mathcal{D} \hat{\mu}_{1}=$ $\prod_{\{\phi\}} \sqrt{N / 2 \pi} d \hat{\mu}_{1}(\phi)$. The last step consists in calculating the normalization factor $\mathcal{A}_{N}$ from eq. (2)

$$
\begin{aligned}
\mathcal{A}_{N} & =\exp [N(-c+c \ln c-\ln c !)] \\
& \times \exp \left[\frac{c}{2}+\frac{1}{4}-\frac{1}{2} \ln 2+O\left(\frac{1}{N}\right)\right] .
\end{aligned}
$$

Substituting eq. (A7) in eq. (A6) and making the following change of the integration variable

$$
\hat{\mu}_{1}(\phi)=i c \int d \boldsymbol{\psi} U(\phi, \psi) \Psi(\boldsymbol{\psi}),
$$

one can rewrite $\left\langle\left[Z_{N}(z)\right]^{n}\right\rangle$ as in eq. (9).

\section{Appendix B: Correspondence with rigorous results}

The main rigorous result of reference [20] is the following theorem:

Fix $c \geq 3$ and let $G_{N}$ be a random c-regular graph on $N$ vertices with adjacency matrix $A_{N}$. Let $\lambda_{1} \geq \cdots \geq \lambda_{N}$ be the eigenvalues of $(c-1)^{-1 / 2} A_{N}$.

Suppose that $f$ is a function defined on the complex plane, analytic inside a Bernstein ellipse of radius $2 \rho$, where $\rho=(c-1)^{\alpha}$ for some $\alpha>3 / 2$, and such that $|f(z)|$ is bounded inside the ellipse. Then $f(x)$ can be expanded on $[-2,2]$ as

$$
f(x)=\sum_{k=0}^{\infty} a_{k} \Gamma_{k}(x),
$$

and $Y_{f}^{(N)}=\sum_{i=1}^{N} f\left(\lambda_{i}\right)-N a_{0}$ converges in law as $N \rightarrow$ $\infty$ to the infinitely divisible random variable

$$
Y_{f}=\sum_{k=1}^{\infty} \frac{a_{k}}{(c-1)^{k / 2}} C N B W_{k}^{(\infty)} .
$$

Let us specify the important quantities that appear in this theorem. The polynomials $\Gamma_{k}(x)$ are defined according to

$$
\begin{aligned}
\Gamma_{0}(x) & =1, \\
\Gamma_{2 k}(x) & =2 T_{2 k}\left(\frac{x}{2}\right)+\frac{c-2}{(c-1)^{k}} \quad k \geq 1, \\
\Gamma_{2 k+1}(x) & =2 T_{2 k+1}\left(\frac{x}{2}\right) \quad k \geq 0,
\end{aligned}
$$

where $T_{k}(x)$ are Chebyshev polynomials of the first kind, which fulfill the orthogonality relations

$$
\int_{-1}^{1} \frac{d x}{\sqrt{1-x^{2}}} T_{i}(x) T_{j}(x)=\left\{\begin{array}{ll}
0 & \text { if } i \neq j \\
\pi & \text { if } i=j=0 \\
\frac{\pi}{2} & \text { if } i=j \neq 0
\end{array} .\right.
$$

The random variable $C N B W_{k}^{(\infty)}$ is the number of cyclically non-backtracking walks of length $k$ in $G_{N}$ [20]. It has the explicit form

$$
C N B W_{k}^{(\infty)}=\sum_{j \mid k} 2 j C_{j}^{(\infty)}
$$

where the sum runs over the values $j=3, \ldots, \infty$ such that $\frac{k}{j}$ is an integer. The variables $C_{j}^{(\infty)}$ are independent Poisson random numbers with average $(c-1)^{j} / 2 j$.

The above theorem makes a statement about the deviation of a general linear functional of the eigenvalues, defined by $\sum_{i=1}^{N} f\left(\lambda_{i}\right)$, with respect to the quantity $N a_{0}$, as $N$ grows to infinity. It tells us that the deviation $\sum_{i=1}^{N} f\left(\lambda_{i}\right)-N a_{0}$ converges in distribution to a nonGaussian random variable $Y_{f}=O(1)$, defined in eq. (B2). Hence we can write down the following equation for the ensemble average of the linear functional

$$
\frac{1}{N}\left\langle\sum_{i=1}^{N} f\left(\lambda_{i}\right)\right\rangle=a_{0}+\frac{1}{N} \sum_{k=1}^{\infty} \frac{a_{k}}{(c-1)^{k / 2}} V_{k}
$$

where

$$
V_{k}=\sum_{j \mid k}(c-1)^{j}
$$

The right hand side of eq. (B8) has been obtained by taking the average over the Poisson random variables present in $C N B W_{k}^{(\infty)}$. Note also that $V_{1}=V_{2}=0$, because the sum over $j$ in the definition of $V_{k}$ starts at $j=3$.

We have computed the $O(1 / N)$ correction to the averaged resolvent

$$
R_{N}(z)=\frac{1}{N}\langle\operatorname{Tr} \boldsymbol{G}(z)\rangle=\frac{1}{N}\left\langle\sum_{i=1}^{N} \frac{1}{z-\lambda_{i}}\right\rangle,
$$

with $z=\lambda-i \eta$. Thus, $R_{N}(z)$ is the ensemble average of a linear functional of the form $N^{-1} \sum_{i=1}^{N} f\left(\lambda_{i}\right)$, with $f\left(\lambda_{i}\right)=1 /\left(z-\lambda_{i}\right)$. From eq. (B8), we expect that $a_{0}$ gives the leading behavior of $R_{N}(z)$, while the other coefficients $a_{1}, \ldots, a_{\infty}$ contain information about the $O(1 / N)$ fluctuations. Thus, the computation boils down to determine $a_{0}, \ldots, a_{\infty}$.

From eq. (B1), we can write

$f(2 x)=a_{0} \Gamma_{0}(2 x)+\sum_{k=1}^{\infty} a_{2 k} \Gamma_{2 k}(2 x)+\sum_{k=0}^{\infty} a_{2 k+1} \Gamma_{2 k+1}(2 x)$.

By multiplying both sides by $\Gamma_{j}(x) / \sqrt{1-x^{2}}$, integrating over $x$ and using eqs. (B3) B5) and (B6), one derives the 
following expressions for the coefficients

$$
\begin{aligned}
a_{0} & =\frac{1}{\pi} \int_{-1}^{1} \frac{d x}{\sqrt{1-x^{2}}} f(2 x)\left[1-(c-2) \sum_{j=1}^{\infty} \frac{T_{2 j}(x)}{(c-1)^{j}}\right] \\
a_{2 k+1} & =\frac{1}{\pi} \int_{-1}^{1} \frac{d x}{\sqrt{1-x^{2}}} T_{2 k+1}(x) f(2 x) \quad k \geq 0 \\
a_{2 k} & =\frac{1}{\pi} \int_{-1}^{1} \frac{d x}{\sqrt{1-x^{2}}} T_{2 k}(x) f(2 x) \quad k \geq 1
\end{aligned}
$$

There are many ways to write down an explicit form for the Chebyshev polynomials. Here we use the following expression

$$
T_{j}(x)=\frac{1}{2}\left[x+i \sqrt{1-x^{2}}\right]^{j}+\frac{1}{2}\left[x-i \sqrt{1-x^{2}}\right]^{j},
$$

valid in the domain $x \in[-1,1]$. By substituting eq. (B15) in eq. (B12), we get

$$
a_{0}=\frac{2 c(c-1)}{\pi} \int_{-1}^{1} d x f(2 x) \frac{\sqrt{1-x^{2}}}{\left[c^{2}-4 x^{2}(c-1)\right]} .
$$

The change of integration variables

$$
x=\frac{\lambda}{2 \sqrt{c-1}},
$$

leads to

$$
a_{0}=\int_{-2 \sqrt{c-1}}^{2 \sqrt{c-1}} d \lambda f\left(\frac{\lambda}{\sqrt{c-1}}\right) \rho_{0}(\lambda),
$$

where $\rho_{0}(\lambda)$ is the leading contribution to the eigenvalue distribution, as presented in eq. (35). We do not need to compute explicitly the above integral, since eq. B18 is the Stieltjes transform of the eigenvalue distribution $\rho_{0}(\lambda)$ : this is nothing more than the definition of the averaged resolvent. In order to evaluate the $O(1 / N)$ correction, we need to write down the coefficients $a_{1}, \ldots, a_{\infty}$ in the same integral form.

By making the change of variables (B17) in eqs. (B13) and (B14), we get

$$
\begin{gathered}
a_{2 j+1}=\int_{-2 \sqrt{c-1}}^{2 \sqrt{c-1}} d \lambda f\left(\frac{\lambda}{\sqrt{c-1}}\right) C(\lambda) T_{2 j+1}\left(\frac{\lambda}{2 \sqrt{c-1}}\right), \\
a_{2 j}=\int_{-2 \sqrt{c-1}}^{2 \sqrt{c-1}} d \lambda f\left(\frac{\lambda}{\sqrt{c-1}}\right) C(\lambda) T_{2 j}\left(\frac{\lambda}{2 \sqrt{c-1}}\right),
\end{gathered}
$$

with $C(\lambda)$ defined by eq. (58). Now it is convenient to introduce, for $|\lambda|<2 \sqrt{c-1}$, the function $g_{c}(\lambda)$

$$
g_{c}(\lambda)=\frac{1}{2(c-1)}\left(\lambda+i \sqrt{4(c-1)-\lambda^{2}}\right),
$$

which allows to rewrite, using eq. (B15), the Chebyshev polynomials as follows

$$
T_{j}\left(\frac{\lambda}{2 \sqrt{c-1}}\right)=(c-1)^{j / 2} \operatorname{Re}\left[g_{c}(\lambda)\right]^{j} .
$$

Substituting this form of $T_{j}$ in eqs. (B19) and (B20), and then inserting the resulting expressions in eq. (B8), we obtain

$$
\begin{aligned}
\frac{1}{N}\left\langle\sum_{i=1}^{N} f\left(\lambda_{i}\right)\right\rangle & =\int_{-2 \sqrt{c-1}}^{2 \sqrt{c-1}} d \lambda f\left(\frac{\lambda}{\sqrt{c-1}}\right) \\
& \times\left[\rho_{0}(\lambda)+\frac{1}{N} \rho_{1}(\lambda)\right],
\end{aligned}
$$

where

$$
\rho_{1}(\lambda)=C(\lambda) \operatorname{Re}\left[\sum_{L=3}^{\infty} V_{L} g_{c}^{L}\right]
$$

The above summation starts at $L=3$, because $V_{1}$ and $V_{2}$ are zero.

The $O(1 / N)$ contribution in eq. (B24) is the Stieltjes transform of $\rho_{1}(\lambda)$, which yields the finite size correction $R_{1}(z)$ to the averaged resolvent. In order to compare with our results derived through the replica method, we rewrite eq. (57) according to

$$
\rho_{1}(\lambda)=C(\lambda) \operatorname{Re}\left[\sum_{L=3}^{\infty}(c-1)^{L} \sum_{n=0}^{\infty} g_{c}^{L(n+1)}\right] .
$$

By comparing the coefficients $V_{L}$, defined by eq. (B9), with those of the above equation, we conclude that eqs. (B25) and (B26) are the same.
[1] P. R. D. Cvetković and S. Simić, An introduction to the theory of graph spectra (Cambridge Univ. Press, Cam- bridge, 2010).

[2] A. Cavagna, I. Giardina, and G. Parisi, 
Phys. Rev. Lett. 83, 108 (1999), URL http://link.aps.org/doi/10.1103/PhysRevLett.83.108

[3] R. Abou-Chacra, D. J. Thouless, and P. W. Anderson, Journal of Physics C: Solid State Physics 6, 1734 (1973), URL http://stacks.iop.org/0022-3719/6/i=10/a=009

[4] R. Baxter, Exactly Solved Models in Statistical Mechanics (Academic Press, 1982), ISBN 9780120831821, URL http://books.google.it/books?id=u_JkQgAACAAJ

[5] T. Rogers, PhD thesis, King's College, London (2010).

[6] Y. Kabashima, H. Takahashi, and O. Watanabe, Journal of Physics: Conference Series 233, 012001 (2010), URL http://stacks.iop.org/1742-6596/233/i=1/a=012001

[7] I. Dumitriu and S. Pal, Ann. Probab. 40, 1861 (2012).

[8] H. Kesten, Trans. Amer. Math. Soc. 92, 336 (1959).

[9] B. D. McKay, Linear Algebra Appl. 40, 203 (1981).

[10] K. B. Efetov, Physica A 167, 119 (1990).

[11] A. Montanari and T. Rizzo, Journal of Statistical Mechanics: Theory and Experiment 2005, P10011 (2005), URL http://stacks.iop.org/1742-5468/2005/i=10/a=P10011

[12] G. Parisi and F. Slanina, Journal of Statistical Mechanics: Theory and Experiment 2006, L02003 (2006), URL http://stacks.iop.org/1742-5468/2006/i=02/a=L02003.

[13] V. E. Sacksteder, Phys. Rev. D 76, 105032 (2007), URL http://link.aps.org/doi/10.1103/PhysRevD.76.105032

[14] S. F. Edwards and R. C. Jones, Journal of Physics A: Mathematical and General 9, 1595 (1976), URL http://stacks.iop.org/0305-4470/9/i=10/a=011.

[15] G. J. Rodgers and A. J. Bray, Phys. Rev. B 37, $3557 \quad$ (1988), URL http://link.aps.org/doi/10.1103/PhysRevB.37.3557

[16] U. Ferrari, C. Lucibello, F. Morone, G. Parisi, F. Ricci-Tersenghi, and T. Rizzo, Phys. Rev. B 88, 184201 (2013), URL http://link.aps.org/doi/10.1103/PhysRevB.88.184201

[17] C. Lucibello, F. Morone, G. Parisi, F. Ricci-Tersenghi, and T. Rizzo, Phys. Rev. E 90, 012146 (2014), URL http://link.aps.org/doi/10.1103/PhysRevE.90.012146

[18] G. B. Arous and K. Dang, arXiv:1106.2108 (2011).

[19] I. Dumitriu, T. Johnson, S. Pal, and E. Paquette, Probability Theory and Related Fields 156, 921 (2013), ISSN 0178-8051, URL http://dx.doi.org/10.1007/s00440-012-0447-y.

[20] T. Johnson, arXiv:1112.0704 (2012).

[21] D. S. Dean, Journal of Physics A: Mathematical and General 35, L153 (2002), URL http://stacks . iop.org/0305-4470/35/i=12/a=101

[22] R. Kühn, J. Phys. A: Math. Theor. 41, 295002 (2008).

[23] G. Ergün and R. Kühn, Journal of Physics A: Mathematical and Theoretical 42, 395001 (2009), URL http://stacks.iop.org/1751-8121/42/i=39/a=395001

[24] T. Rogers, C. P. Vicente, K. Takeda, and I. P. Castillo, Journal of Physics A: Mathematical and Theoretical 43, 195002 (2010), URL http://stacks.iop.org/1751-8121/43/i=19/a=195002

[25] R. Kühn and J. van Mourik, Journal of Physics A: Mathematical and Theoretical 44, 165205 (2011), URL http://stacks.iop.org/1751-8121/44/i=16/a=165205

[26] T. Nagao, Journal of Physics A: Mathematical and Theoretical 46, 065003 (2013), URL http://stacks.iop.org/1751-8121/46/i=6/a=065003.

[27] C. Bordenave and M. Lelarge, Random Structures \& Algorithms 37, 332 (2010), ISSN 1098-2418, URL http://dx.doi.org/10.1002/rsa.20313

[28] F. L. Metz, I. Neri, and D. Bollé, Phys. Rev. E 82, 031135 (2010), URL http://link.aps.org/doi/10.1103/PhysRevE.82.031135.

[29] E. N. Economou, Green's functions in quantum physics (Springer, Heidelberg, 2006).

[30] B. Bollobás, European Journal of Combinatorics 1, 311 (1980).

[31] N. C. Wormald, Journal of Combinatorial Theory Series B 31, 168 (1981).

[32] P. J. Davis and P. Rabinowitz, Methods of Numerical Integration (Academic Press, London, 1984).

[33] J. Waldvogel, Towards a General Error Theory of the Trapezoidal Rule in Approximation and Computation, vol. 42 (Springer New York, 2011).

[34] L. N. Trefethen and J. A. C. Weideman, SIAM Review 56, 385 (2014).

[35] G. Hardy, Divergent Series, Chelsea Publishing Series (American Mathematical Society, 1991), ISBN 9780821826492, URL http://books.google.it/books?id=jPccoUKsLdQC

[36] A. Steger and N. C. Wormald, Comb. Probab. Comput. 8, 377 (1999), ISSN 0963-5483, URL http://dx.doi.org/10.1017/S0963548399003867.

[37] J. J. M. Verbaarschot and M. R. Zirnbauer, Ann.Phys. 158, 78 (1984).

[38] G. S. Dhesi and R. C. Jones, Journal of Physics A: Mathematical and General 23, 5577 (1990), URL http: //stacks. iop.org/0305-4470/23/i=23/a=029.

[39] A. D. Mirlin and Y. V. Fyodorov, Journal of Physics A: Mathematical and General 24, 2273 (1991), URL http://stacks.iop.org/0305-4470/24/i=10/a=016

[40] For $z=\lambda<0$ and $|\lambda| \geq\left|\lambda_{b}\right|$, we need to make the replacement $\sqrt{\lambda^{2}-\lambda_{b}^{2}} \rightarrow-\sqrt{\lambda^{2}-\lambda_{b}^{2}}$ in order to obtain that $g_{c} \rightarrow 0$ for $\lambda \rightarrow-\infty$ and, consequently, derive the correct behavior of $R_{0}(\lambda)$ and $R_{1}(\lambda)$ in this regime.

[41] We notice en passant that the matrix elements of the Green function at two points separated by a distance $r>0$ are given by $A\left(g_{c}\right)^{r}$, with an appropriate value of the constant $A$. Therefore, the quantity $g_{c}$, namely the diagonal part of the Green function on the cavity graph, is a key quantity of the model: it controls both the large distance decay of the Green function in the limit $N \rightarrow \infty$ and the $1 / N$ corrections to the resolvent. 